\title{
I. Grundlagen
}




\title{
Aufgaben und Handlungsspielräume der Kommunen als Ort der Inklusion
}

\author{
Ulrich Becker
}

$\begin{array}{ll}\text { I. Einleitung } & 11\end{array}$

II. Kommunale Verantwortung für die Inklusion $\quad 13$

1. Zuständigkeiten: Von den Kommunen zum Staat und zurück?

2. Aufgabenfelder: Kommunale Aufgaben $\quad 15$

a) Sozialrechtliche Aufgaben $\quad 15$

b) Sozialraumorientierung $\quad 17$

c) Quer liegende Direktiven und allgemeine Aufgaben 18

III. Bedingungen kommunalen Handelns in der föderalen Ordnung 19

1. Grenzen der Selbstverwaltung 19

a) Unitarische gesetzliche Vorgaben im Sozialstaat 19

b) Zersplitterung kommunaler Aufgabenwahrnehmung 20

2. Die Finanzierung sozialstaatlicher Aufgaben 21

a) Grundsätze $\quad 21$

b) Aufgabenübertragungsverbot und Konnexitätsprinzip 23

$\begin{array}{ll}\text { IV. Schluß } & 24\end{array}$

\section{Einleitung}

1. Ereignisse haben ihre Zeit, und sie haben ihren Raum. In der bildreichen englischen Sprache heißt es ,events take place“. Diese an sich simple, wenn auch nicht banale Feststellung hat lange in der Sozialpolitik wenig Beachtung gefunden. Deren räumlicher Aspekt spielte kaum eine Rolle. Das hat sich grundlegend geändert, und die Karriere des Begriffs "Sozialraum"1 verdankt sich vielleicht auch dem Umstand, daß er aus weitgehend unbearbeitetem Grund aufgestiegen ist - womit nicht gleichzeitig etwas gegen dessen Bedeutung gesagt ist. Die Notwendigkeit, die räumlichen Bezüge sozialpolitischer Anliegen und Maßnahmen in den Blick zu nehmen, steht vielmehr außer Zweifel.

Welche Analysemöglichkeiten die Raumbezogenheit der Sozialpolitik eröffnet, hängt von dem Raumbegriff ebenso ab wie von der Bedeutung räumlich bedingter Vo-

1 Dazu etwa Jordan, Sozialraumorientierung in der Kinder- und Jugendhilfe, in: Kreft/Mielenz/Trauernicht/Jordan (Hrsg.), Fortschritt durch Recht, Festschrift für Johannes Münder, 2004, S. 255 ff. 
raussetzungen der Interaktion und der personalen Entfaltung. Aus juristischer Perspektive ist der räumliche Bezug aus zwei Gründen aufschlußreich.

Zum einen steht hinter ihm die Ortsgebundenheit hoheitlicher Gewalt: Diese Gewalt ist im Hinblick auf ihren Ausgangspunkt, die Verleihung in Verbindung mit ihrer Legitimation, wie auch im Hinblick auf ihre Wirkung räumlich gegliedert und auf mehrere Ebenen verteilt. Insofern kann von räumlich gestuften Rechtsordnungen gesprochen werden, die zumeist, wenn auch auf der Ebene des Völkerrechts mit (noch) eingeschränkter Bedeutung, ${ }^{2}$ politische Gemeinschaften konstituieren. Die Zuordnung von Kompetenzen zu diesen Ebenen und das Verhältnis des auf der jeweiligen Ebene gesetzten Rechts zueinander sind seit jeher ein klassisches Feld rechtswissenschaftlicher Betätigung.

Zum anderen läßt der Raumbezug zugleich eine alternative, in der Regel dominierende Gliederung in den Hintergrund treten, nämlich die nach sozialpolitischen Sachmaterien. ${ }^{3}$ Das ist deshalb vielfach weiterführend, weil eine Ordnung nach Materien an mehr oder weniger historisch zufällig entstandenen Institutionen hängt und deshalb Gefahr läuft, Zusammenhängendes nicht in den Blick zu nehmen. Diese Gefahr wird heute verstärkt wahrgenommen, ${ }^{4}$ weil sozialpolitische Ziele neu formuliert und übergreifender verwirklicht werden müssen, wofür die Inklusion von behinderten Menschen ein besonders prominentes Beispiel darstellt. Hingegen ist der Raum, auch wenn er keineswegs als feste vorgegebene Größe angesehen werden kann, sondern je nach relevanter Fragestellung differiert und oft auch erst durch die jeweils Handelnden und die dabei relevanten Handlungszusammenhänge geschaffen wird, doch immerhin auch ein von außen beschreibbarer allgemeiner Bezugspunkt.

2. Damit ist über die Begrenzung des Raums noch nichts gesagt. Die Kommune bei unserer Tagung und deshalb auch den folgenden Ausführungen in den Mittelpunkt einer raumbezogenen Betrachtung zu stellen, hat gute Gründe. Lange Zeit weitgehend unbeachtet, gewinnt sie im Zuge neuerer sozialpolitischer Entwicklungen an Bedeutung. Das soll in einem ersten Schritt, zusammen mit den Gründen für diese Aufwertung, kurz

2 Vgl. aber auch Uerpmann, Die Europäische Menschenrechtskonvention und die deutsche Rechtsprechung - Ein Beitrag zum Thema Völkerrecht und Landesrecht, 1993; ders., Internationales Verfassungsrecht, JZ 2001, S. 565 ff.; Pfeffer, Das Verhältnis von Völkerrecht und Landesrecht - Eine kritische Betrachtung alter und neuer Lehren unter besonderer Berücksichtigung der Europäischen Menschenrechtskonvention, 2009.

3 Klassisch und mit internationaler Bedeutung das Abkommen Nr. 102 der IAO vom 28. Juni 1952 über die Mindestnormen der Sozialen Sicherheit (BGBl. II 1957, S. 1321, 1322; abrufbar auch unter http://www.ilo.org/ilolex/german/docs/convdisp1.htm).

4 Vgl. nur Becker, Sozialrecht und Sozialrechtswissenschaft, ZÖR 65 (2010), S. 607, 618 ff.; ders., Das gegliederte System der sozialen Sicherung - bietet die Rechtsvergleichung eine Anleitung zur Vereinfachung?, NDV 2010, S. 510 ff.; vgl. zu den sog. „New Social Risks“ Taylor-Gooby (Hrsg.), New risks, new welfare: the transformation of the European welfare state, 2004; Bonoli, The Politics of the New Social Policies: Providing Coverage against New Social Risks in Mature Welfare States, Policy and Politics 33 (2005), S. $431 \mathrm{ff}$. 
dargelegt werden (II.). Hintergrund ist der Umstand, daß das Soziale auch in gemeinschaftlichen Näheverhältnissen aufgehoben sein kann und die Kommunen ein flächendeckendes wie universell einsetzbares Netz politischer Gemeinschaften darstellen - im übrigen auch in anderen Ländern, in denen sie dementsprechend ebenfalls sozialpolitische Aufgaben wahrnehmen, etwa die local communities in England für die Zurverfügungstellung von social services. ${ }^{5}$ In Deutschland wird die kommunale Aufgabenwahrnehmung in einen mehrfach geteilten föderalen Rahmen gestellt. Die den Kommunen eingeräumte Selbstverwaltung ist zwar eine rechtliche Garantie für Handlungsoptionen, die aber begrenzt ist: unmittelbar durch gesetzliche Vorgaben, mittelbar und dennoch nicht weniger wirksam durch finanzielle Vorgaben. Beides wird in einem zweiten Schritt behandelt (III.), der als allgemeine Einführung in die rechtlich geformten Bedingungen des kommunalen Handelns dienen soll.

\section{Kommunale Verantwortung für die Inklusion}

\section{Zuständigkeiten: Von den Kommunen zum Staat und zurück?}

Seit der Herausbildung der Städte als eigenen Regeln folgende politische Gemeinschaften - in denen Leibeigenschaft durch Bürgerrechte abgelöst wird, wenn auch nicht ohne Etablierung einer neuen herrschenden Einwohnerschicht - übernahmen diese auch sozialpolitische Aufgaben. Die Errichtung kommunaler Spitäler, die verschiedene Funktionen erfüllten, wofür etwa die Heilig-Geist-Spitäler mit dem berühmten HeiligenGeist-Hospital in Lübeck oder die zum Teil noch heute existierenden Bürgerspitale stehen, aber auch frühe städtische Vorschriften über die Bekämpfung von unbotmäßigem Verhalten 6 legen davon Zeugnis ab.

Schon mit der Konfessionsspaltung und der ersten Säkularisierungswelle verlagerten sich die Verantwortlichkeiten zur Lösung sozialer Probleme von der gesellschaftlichen hin zur gemeinschaftlichen Sphäre, vor allem im Schul- und Armenwesen. ${ }^{7}$ Mit der Herausbildung der modernen Nationalstaaten erfuhr diese Vergemeinschaftung eine staatliche Überformung. Das preußische ALR bringt das zum Ausdruck, wenn es dort heißt: „Dem Staate kommt es zu, für die Ernährung und Verpflegung derjenigen Bürger zu sorgen, die sich ihren Unterhalt nicht selbst verschaffen, und denselben auch von

5 Vgl. zu Pflegeleistungen näher Landauer, Die staatliche Verantwortung für die stationäre Langzeitpflege in England und Deutschland, 2012, S. $137 \mathrm{ff}$.

6 Das gilt selbst in London, obwohl in den englischen Städten der Königsfriede und nicht ein städtischer Sonderfriede als Grundlage der Ordnung galt, vgl. dazu Rexroth, Das Milieu der Nacht, 1999, S. 67 ff., am Beispiel des Umgangs der Obrigkeit mit Randgruppen im spätmittelalterlichen London.

7 Stolleis, Geschichte des Sozialrechts, in Deutschland, 2003, S. 15 ff. 
andern Privatpersonen, welche nach besonderen Gesetzen dazu verpflichtet sind, nicht erhalten können. " 8 Zwar bleiben die Kommunen hier ebenfalls verpflichtet. ${ }^{9}$ Und die Verpflichtung wird inhaltsreicher: So spricht Hegel in seinen Zusätzen zu den Grundlinien der Philosophie des Rechts, ohne jemals die Gemeinden zu erwähnen, von einer „Polizei“ und meint damit die Schaffung einer lebensbefähigenden Ordnung der bürgerlichen Gesellschaft, deren Inhalte sehr an den viel später von Forsthoff entwickelten und umfassenderen Begriff der Daseinsvorsorge ${ }^{10}$ erinnern. Denn Hegel führt aus: „Die polizeiliche Aufsicht und Vorsorge hat den Zweck, das Individuum mit der allgemeinen Möglichkeit zu vermitteln, die zur Erreichung der individuellen Zwecke vorhanden ist. Sie hat für Straßenbeleuchtung, Brückenbau, Taxation der täglichen Bedürfnisse, so wie für die Gesundheit Sorge zu tragen."11

Jedoch stehen die Kommunen nun unter einer staatlichen Gesamtverantwortung. Damit und mit der nachfolgenden Konstitutionalisierung zunächst auf Landes-, dann auf Bundesebene waren die Grundsteine für eine umfassende staatliche Sozialpolitik gelegt, die im 20. Jahrhundert näher ausformuliert wurde.

Vor diesem Hintergrund ist bemerkenswert, daß seit einigen Jahren von einer „Rekommunalisierung“ die Rede ist - zwar zum Teil bezogen auf die Rückgängigmachung von Privatisierungen der Daseinsvorsorge, ${ }^{12}$ zum Teil aber auch auf die Gewährung von Sozialleistungen. Diese Entwicklung hat ihren Grund in veränderten sozialpolitischen Zielsetzungen, nämlich dem Versuch, stärker auf das Verhalten Einzelner Einfluß zu nehmen. Für diese je nach Standpunkt pädagogischen oder paternalistischen Bestrebungen ${ }^{13}$ stehen die Aktivierungsstrategie, aber auch die immer weiter verfeinerte Bekämpfung von Diskriminierungen. Nun kann man sich darüber streiten, inwieweit sozialpolitische Interventionen darauf gerichtet sein müssen oder dürfen, Teilhabechancen zu eröffnen oder tatsächlich eine Teilhabe zu bewirken. In jedem Fall spielen aber für teilhabebezogene Ansätze soziale Interaktionen im unmittelbaren Umfeld, in räumlicher Nähe, eine besondere Rolle. Die selbstbestimmte Entfaltung wie der Zugang zu existentiell wichtigen Leistungen und Einrichtungen sind nur in konkreten räumlichen Zusammenhängen möglich. Daß Kommunen insofern eine besondere Verantwortung und Gestaltungsaufgabe zukommt, liegt auf der Hand. Sie können aufgrund ihrer

8 19. Titel, § 1, zitiert nach Koch, Allgemeines Landrecht für die preußischen Staaten, Band 4,1, 3. Aufl. 1863.

9 19. Titel, § 10: „Auch Stadt- und Dorfgemeinden müssen für die Ernährung ihrer verarmten Mitglieder und Einwohner sorgen."

10 Forsthoff, Die Verwaltung als Leistungsträger, 1938; ders., Rechtsfragen der leistenden Verwaltung, 1959; zu der Begriffsentfaltung Kersten, Die Entwicklung des Konzepts der Daseinsvorsorge im Werk von Ernst Forsthoff, Der Staat 44 (2005), S. 543 ff.; zu dessen heutiger Bedeutung Krajewski, Rechtsbegriff Daseinsvorsorge?, VerwArch 99 (2008), S. 174 ff.

11 Hegel, Grundlinien der Philosophie des Rechts, in: ders., Sämtliche Werke. Jubiläumsausgabe in zwanzig Bänden. Siebenter Band, hrsg. von Glockner, 4. Aufl. 1964, Zusatz zu § 236.

12 Dazu Bauer, Zukunftsthema „Rekommunalisierung“, DÖV 2012, S. 329 ff.

13 Vgl. Heinig, Paternalismus im Sozialstaat, in: Anderheiden/Bürkli/Heinig/Kirste/Seelmann (Hrsg.), Paternalismus und Recht, 2006, S. $157 \mathrm{ff}$. 
Kenntnisse der örtlichen Gegebenheiten in vielen Fällen zur zunehmend angestrebten Zielgerichtetheit sozialer Leistungen beitragen. Und sie bilden ein flächendeckendes Netz erreichbarer Behörden, das eine umfassende räumliche Abdeckung der Bemühungen um sozialen Schutz aller Einwohner erleichtert.

Das bedeutet nicht ein Zurück zu mittelalterlichen Verhältnissen - auch wenn etwa das Fördern und Fordern, wie es der im SGB II angelegten Aktivierung zugrunde liegt, an eine obrigkeitliche Armenpolitik im Zeichen von ,Zuwendung und Strenge“, wie das Stolleis formuliert hat, ${ }^{14}$ erinnert. Die rechtlichen Rahmenbedingungen der kommunalen Sozialpolitik haben sich vielmehr in zweifacher Weise grundlegend geändert. Erstens beruht diese, bei aller Wiederentdeckung von Eigenverantwortung und der damit auch verbundenen Vorstellung, die Gemeinschaften funktionsfähig zu halten, ${ }^{15}$ auf einer menschenrechtlichen Basis: Jeder Einzelne besitzt durchsetzbare Ansprüche auf sozialen Schutz, die nicht von einem sittlichen Wohlverhalten, sondern höchstens von gesetzlich festgelegten und mit dem Schutzzweck vereinbaren Voraussetzungen abhängen. Zweitens sind die Kommunen in eine gesamtstaatliche und zunehmend sogar EUweite Verantwortung eingebunden, ${ }^{16}$ die ihnen rechtlich verbindliche Vorgaben auferlegt und zu einer zunehmenden rechtlichen Verflechtung zwischen den politischen Ebenen führt.

\section{Aufgabenfelder: Kommunale Aufgaben}

\section{a) Sozialrechtliche Aufgaben}

Bevor auf diese Verflechtung zurückzukommen ist, soll ein Blick auf die für die Themenstellung relevanten kommunalen Aufgaben und ihre jüngsten Veränderungen geworfen werden, beginnend mit denen, die aus dem formalen Sozialrecht abzuleiten sind. In diesem Zusammenhang läßt sich die These von der wachsenden sozialpolitischen Bedeutung der Kommunen sowohl in quantitativer als auch in qualitativer Hinsicht belegen, wenn auch hier nur in Stichworten.

14 Stolleis, Geschichte des Sozialrechts (Fußn. 7), S. 17.

15 Becker, Sozialmodell und Menschenbild in der „Hartz-IV” Gesetzgebung, in: Behrends/Schumann (Hrsg.), Gesetzgebung, Menschenbild und Sozialmodell im Familien- und Sozialrecht, Abhandlungen der Akademie der Wissenschaften zu Göttingen, 2008, S. 39 ff.; grds. Zacher, Grundtypen des Sozialrechts, in: Fürst/Herzog/Umbach (Hrsg.), FS für Zeidler, 1987, S. 571, 572.

16 Vgl. Art. 4 Abs. 2 S. 1 EUV; Art. 5 Abs. 3 EUV; Art. 170 Abs. 1 AEUV; Art. 300 AEUV; Protokoll Nr. 26 des AEUV über Dienste von allgemeinem Interesse. Aus dem Schrifttum: Knauff, Die Kommunen als Träger der Daseinsvorsorge, WiVerw 2011, S. 79 ff.; Schmidt-Leithoff, Gemeindewirtschaft im Wettbewerb, 2011; Burgi, Kommunalrecht, 3. Aufl. 2010, § 4 Kommunen und Europarecht; Pfisterer, Kommunale Selbstverwaltung und lokale Governance vor dem Hintergrund des europäischen Integrationsprozesses, 2009; Bergmann, Deutsche Kommunen und Europa, BWGZ 2009, S. 300 ff. 
aa) Mit dem verstärkten Bekenntnis zu Inklusion und Teilhabe von Menschen mit Behinderung sind neue Querschnittsaufgaben entstanden, und das nicht erst durch Inkrafttreten des SGB IX im Jahre 2001. Zu nennen sind hier die Eingliederungshilfe für behinderte Menschen gem. $\S \S 53 \mathrm{ff}$. SGB XII sowie die Eingliederungshilfe für seelisch behinderte Kinder und Jugendliche gem. § 35a SGB VIII ${ }^{17}$, wobei es dem Gesetzgeber bislang nicht gelungen ist, die Schwierigkeiten, die hinsichtlich der Abgrenzung dieser beiden Formen von Hilfe für junge Menschen mit Behinderung entstehen, zu beseitigen. 18

Auch die bereits angesprochene aktivierende Arbeitsmarktpolitik bringt hinsichtlich der Eingliederung von Menschen mit Behinderung neue Aufgaben für die Kommunen. Sie ist schon nach $\S 1$ Abs. 2 S. 3 Nr. 5 SGB II auf die Überwindung von behindertenspezifischen Nachteilen auszurichten, was bei den Eingliederungsleistungen gem. $§ 16$ Abs. 1 S. 2 SGB II zu beachten ist. Dabei fordert nun $\S 16$ a SGB II ${ }^{19}$, zur Verwirklichung einer ganzheitlichen und umfassenden Betreuung und Unterstützung bei der Eingliederung in Arbeit auch die Betreuung minderjähriger oder behinderter Kinder oder die häusliche Pflege von Angehörigen vorzusehen. Zudem wird gem. § 21 Abs. 4 S. 1 SGB II ein Mehrbedarf von erwerbsfähigen behinderten Menschen im Rahmen der Grundsicherung anerkannt.

bb) Gleichzeitig sind die Anforderungen an bereits bestehende Aufgaben weiter erhöht worden, was sich besonders gut in der Kinder- und Jugendhilfe beobachten läßt, einer Kernmaterie kommunaler Sozialpolitik, die nach Lorenz von Stein in ,der polizeilichen Epoche“ als „Sorge der Verwaltung für das Leben der geborenen Kinder“ zu einer öffentlichen Aufgabe wurde. ${ }^{20}$

Seit Inkrafttreten des SGB VIII im Jahre 1990 gab es zahlreiche Änderungen, die unter Kindesschutz-21, aber auch Qualitätsaspekten ${ }^{22}$ die Aufgabenerfüllung anspruchs-

17 Jüngst zur Begriffsproblematik der ,seelischen Behinderung“ i.S.v. § 35a SGB VIII Riehle, Anmerkungen zu § 35a SGB VIII, ZFSH/SGB 2012, S. $207 \mathrm{ff}$.

18 Dazu Pitschas, Behinderte Menschen in der kommunalen Sozialpolitik, SGb 2009, S. 253, 259 f.; Banafsche, Kinder und Jugendliche mit Behinderung zwischen SGB VIII und SGB XII - im Lichte der UN-Behindertenrechtskonvention, ZKJ 2011, S. 116 ff.; Gerlach/Hinrichs, Inklusion und die „Große Lösung“ für die Jugend- und Behindertenhilfe, ZKJ 2012, S. 86 ff. (Teil 1), 130 ff. (Teil 2).

19 Eingefügt mit Wirkung zum 1.1.2009 durch das Gesetz zur Neuausrichtung der arbeitsmarktpolitischen Instrumente (ArbMINAG) v. 21.12.2008, BGB1. I 2008, S. 2859.

20 Lorenz v. Stein, Die Verwaltungslehre, Teil 2: Wirkliche Innere Verwaltung, 2. Neudruck der Ausgabe Stuttgart 1866, 1975, S. 162.

21 Etwa durch Einfügung des § 8a in das SGB VIII mit Wirkung zum 1.10.2005 durch das Gesetz zur Weiterentwicklung der Kinder- und Jugendhilfe - KICK: Kinder- und Jugendhilfeweiterentwicklungsgesetz, verkündet am 27.12.2004, BGB1. I 2004, S. 3852., der bei Anzeichen einer Kindeswohlgefährdung umfassende Mitteilungs- und Dokumentationspflichten des Jugendamtes aber auch der freien Träger vorsieht oder durch Inkrafttreten des Gesetzes zur Stärkung eines aktiven Schutzes von Kindern und Jugendlichen (Bundeskinderschutzgesetz - BKiSchG) v. 22.12.2011 (BGBl. I 2011, S. 2975), das unter anderem zur Sicherstellung von persönlicher Zuverlässigkeit sämtlicher in der Jugendhilfe tätigen Personen inklusive Ehrenamtlicher die Vorlage eines Führungszeugnisses 
voller werden lassen. Kommunen als Leistungsträger sind im Rahmen ihrer als Gesamtverantwortung ( $\$ 79$ SGB VIII) bezeichneten Gewährleistungsverantwortung bei der Aufsicht und Steuerung freier Träger mit höheren Anforderungen konfrontiert. Hinzuweisen ist neben der Planungsverantwortung ( $\$ 80$ SGB VIII) auf die durch das BKiSchG neu eingefügte Aufgabe der Qualitätsentwicklung (§ 79a SGB VIII). Ferner bringt der mit dem Kinderförderungsgesetz (KiföG) ${ }^{23}$ in Gang gesetzte Ausbau institutionalisierter Kinderbetreuung 24 neue Herausforderungen für die Kommune, und zwar sowohl in deren Rolle als Leistungsträger, weil die neuen entstehenden Kindertageseinrichtungen auch beaufsichtigt werden wollen ${ }^{25}$, aber auch als Leistungserbringer, weil Kitas zu einem großen Teil kommunale Einrichtungen sind - und demnächst, d.h. ab dem 1.8.2013, werden sich die Kommunen den Rechtsansprüchen auf Kinderkrippenplätze ausgesetzt sehen.

\section{b) Sozialraumorientierung}

Die erwähnte Planungsverantwortung in der Jugendhilfe enthält zugleich einen der wenigen normativen Anknüpfungspunkte für eine Sozialraumorientierung kommunaler Sozialpolitik. Er kommt zum Ausdruck durch die Formulierung „Einrichtungen und Dienste sollen so geplant werden, daß insbesondere Kontakte [...] im sozialen Umfeld gepflegt und erhalten werden können“ (§ 80 Abs. 2 Nr. 1 SGB VIII). Ähnliche Bezugnahmen finden sich in den Einleitungsvorschriften des SGB VIII, nämlich auf die „Umwelt" (§ 1 Abs. 3 S. 4 SGB VIII), sowie im Zusammenhang mit den Hilfen zur Erziehung, die das „soziale Umfeld des Kindes oder des Jugendlichen“ einbeziehen sollen (§ 27 Abs. 2 S. 2 Hs. 2 SGB VIII).

Die rechtliche Bedeutung dieser räumlichen Orientierung bleibt allerdings fraglich. ${ }^{26}$ Sie enthält nach der Rechtsprechung und dem Schrifttum jedenfalls keine Rechtsgrundlage für eine budgetierende Finanzierung von Leistungserbringern. ${ }^{27}$ Eine klare gesetz-

gem. $\S \S 30,30$ a BZRG beim jeweiligen Träger der öffentlichen Jugendhilfe in regelmäßigen Abständen vorsieht, vgl. dazu Weber, Das erweiterte Führungszeugnis als Instrument des Kinderschutzes, JAmt 2012, S. 62 ff.

22 Etwa durch mit Einführung der $\S \S 78$ a ff. SGB VIII durch das Zweite Gesetz zur Änderung des Elften Buches Sozialgesetzbuch und anderer Gesetze vom 29.5.1998, BGBl. I 1998, S. 1188; vgl. allgemein zu Qualitätsaspekten im Sozialrecht die Beiträge in SDSRV 61 (2012) sowie Becker/Meeßen/Neueder/Schlegelmilch/Schön/Vilaclara, Strukturen und Prinzipien der Leistungserbringung im Sozialrecht - Teil 2, VSSR 2012, S. 1, 21 ff.

23 Gesetz zur Förderung von Kindern unter drei Jahren in Tageseinrichtungen und in Kindertagespflege (Kinderförderungsgesetz - KiföG), BGBl. I 2008, S. 2403.

24 Vgl. 7. Familienbericht der Bundesregierung, BT-Drucks. 16/1360, S. 54 ff., zu den infrastrukturellen Komponenten einer zeitgemäßen Familienpolitik.

25 So schreibt $\S 45$ SGB VIII auch eine Betriebserlaubnis für Kitas vor.

26 Dazu Nellissen, Sozialraumorientierung im aktivierenden Sozialstaat, 2006, S. $22 \mathrm{ff}$.

27 So jüngst wieder OVG Nieders. v. 9.7.2010, 4 ME 306/09 = JAmt 2011, 102; dazu Münder, Wieder einmal: Sozialraumorientierung auf dem rechtlichen Prüfstand, JAmt 2011, S. 69 ff.; ebenso Wall- 
liche Grundlage wäre aber angesichts des Grundrechts der Berufsfreiheit der Leistungserbringer ${ }^{28}$ sowie des Wunsch- und Wahlrechts der Leistungsempfänger ${ }^{29}$ erforderlich, um die mit einer entsprechenden Finanzierung bezweckte Exklusivität zu rechtfertigen.

Das Sozialraumkonzept hat insofern keine unmittelbare Außenwirkung. Es führt zu nicht mehr, aber auch nicht weniger als einer Verpflichtung, das Handeln sämtlicher sozialer Akteure auch auf der lokalen Ebene unter sozialpolitischer Perspektive miteinander abzustimmen. Das immerhin bringt für die Kommunen im Rahmen ihrer Planungsverantwortung eine neue und nicht zu unterschätzende Vernetzungsaufgabe mit sich. 30

\section{c) Quer liegende Direktiven und allgemeine Aufgaben}

Nicht zuletzt weil die Inklusion behinderter Menschen auf verfassungsrechtlichen und vor allem mit der UN-Konvention über die Rechte von Menschen mit Behinderungen ${ }^{31}$ auch auf völkerrechtlichen Direktiven beruht ${ }^{32}$, die noch im einzelnen Gegenstand der nachfolgenden Beiträge sein werden, ist der Blick über das Sozialrecht ${ }^{33}$ hinaus zu weiten.

Zum einen auf andere und eigenständig geregelte Politikbereiche, insbesondere das Schulrecht, das hier zumindest einmal genannt werden soll. Die nie verstummte „Integrationsdebatte“34 ist hier ebenfalls als „Inklusionsdebatte“ neu und stärker als je zuvor entbrannt, was auch in dieser Hinsicht den bemerkenswert starken Einfluß der genann-

rabenstein, Wettbewerb in der Sozialwirtschaft: rechtliche Steuerung der politischen Steuerung, Archiv für Wissenschaft und Praxis der Sozialen Arbeit 2009, S. 36, 41.

28 Zur Berufsfreiheit der Leistungserbringer Becker/Meeßen/Neueder/Schlegelmilch/Schön/Vilaclara, Strukturen und Prinzipien der Leistungserbringung im Sozialrecht - Teil 1, VSSR 2011, S. 323, 343 ff.

29 Zum Individualisierungsprinzip Becker/Meeßen/Neueder/Schlegelmilch/Schön/Vilaclara, Strukturen und Prinzipien der Leistungserbringung im Sozialrecht - Teil 3, VSSR 2012, S. 103, $121 \mathrm{ff}$.

30 Vgl. bspw. das Konzept „REGSAM - Regionale Netzwerke für Soziale Arbeit“ der Landeshauptstadt München, das Rahmenbedingungen für eine Vernetzung von Politik, Verwaltung, Bürgerinnen und Bürgern, Verbänden und sonstigen Akteuren sozialer Arbeit setzt. Informationen abrufbar unter http://www.regsam.net/de. Allgemein zu kommunaler Sozialpolitik als Querschnittsaufgabe und Netzwerkstruktur Pitschas, Kommunale Sozialpolitik, in: v. Maydell/Ruland (Hrsg.), Sozialrechtshandbuch (SRH), 3. Auflage 2003, S. 1295, 1333, Rdnr. 96 ff.

31 Überführung in deutsches Bundesrecht durch das „Gesetz zu dem Übereinkommen der Vereinten Nationen vom 13.12.2006 über die Rechte von Menschen mit Behinderungen sowie zu dem Fakultativprotokoll vom 13.12.2006 zum Übereinkommen der Vereinten Nationen über die Rechte von Menschen mit Behinderungen" vom 21.12.2008 (BGB1. II 2008, S. 1419).

32 Dazu einführend Welti, Rechtliche Voraussetzungen von Barrierefreiheit, NVwZ 2012, S. $725 \mathrm{ff}$.

33 Zur Vereinbarkeit der UN-Behindertenrechtskonvention mit dem deutschen Sozialrecht jüngst $\mathrm{Ba}$ nafsche, Die UN-Behindertenrechtskonvention und das deutsche Sozialrecht, SGb 2012, S. $373 \mathrm{ff}$. (Teil I) und S. $440 \mathrm{ff}$. (Teil II).

34 Vgl. nur Becker/Graser (Hrsg.), Perspektiven der schulischen Integration von Kindern mit Behinderung, 2004. 
ten UN-Behindertenrechtskonvention belegt. Damit sind so viele und vielfältige Fragen aufgeworfen, daß diese einer eigenständigen Behandlung bedürfen und deshalb nicht Gegenstand dieser Tagung sein sollen, auch wenn sie kommunale Schulen betreffen.

Zum anderen und viel allgemeiner kann sich auch im übrigen die angestrebte Inklusion nicht in der Zurverfügungstellung spezieller Leistungen im Sinne eines Ausgleichs spezifischer Nachteile erschöpfen, sondern stellt neben einer Förderung zugleich Anforderungen an die Öffnung der für die Allgemeinheit gedachten Einrichtungen. Das beginnt bei der Benutzung der Straßen und öffentlichen Verkehrsmittel, geht über die Museen, Sportanlagen und Theater bis zur Ermöglichung der Kommunikation. Dabei unterliegt die kommunale Aufgabenerfüllung quer liegenden, auf bestimmte Tätigkeitsfelder bezogenen Konkretisierungen der allgemeinen Vorgaben. Das gilt insbesondere für das Baurecht: Zu nennen sind hier $\S 171$ e BauGB, der unter dem Titel ,soziale Stadt“ Maßnahmen zur Verhinderung von Segregation vorsieht, die allerdings Einzelpersonen nicht unmittelbar zugute kommen, und $\S 1$ Abs. 6 Nr. 3 BauGB, wonach die Bedürfnisse von Menschen mit Behinderung als öffentlicher Belang im Rahmen der Bauleitplanung zu beachten sind. Praktisch noch wichtiger sind die landesrechtlichen Vorschriften zum barrierefreien Bauen wie etwa Art. 48 BayBO. Dessen Abs. 2 betrifft öffentlich zugängliche Anlagen, neben den gemeindlichen Einrichtungen im eigentlichen Sinn gehören dazu auch Verwaltungsgebäude und private Einrichtungen wie Hotels und Gaststätten. Er wird in Bayern - wie in anderen Ländern ${ }^{35}$ - flankiert durch eine gleichstellungspolitische Vorschrift (Art. 10 Abs. 1 S. 1, 2 des Bayerischen Behindertengleichstellungsgesetzes - BayBBG), nach der die Träger öffentlicher Gewalt ihre Neubauten sowie große Um- oder Erweiterungsbauten entsprechend den allgemein anerkannten Regeln der Technik barrierefrei zu gestalten haben. Diese Verpflichtung steht - anders als die des Bauordnungsrechts - nicht unter dem Vorbehalt einer verhältnismäßigen Mehrbelastung.

\section{Bedingungen kommunalen Handelns in der föderalen Ordnung}

\section{Grenzen der Selbstverwaltung}

\section{a) Unitarische gesetzliche Vorgaben im Sozialstaat}

Kommunen sind also sowohl als Sozialleistungsträger wie als Garanten einer weit verstandenen, kulturelle und sportliche Einrichtungen mit umfassenden Daseinsvorsorge gefordert, wenn es um die Inklusion von Menschen mit Behinderungen geht. Grund-

35 Vgl. zu den Texten die Sammlung von Frehe/Welti (Hrsg.), Behindertengleichstellungsrecht - Textsammlung mit Einführungen, 2010. 
sätzlich können sie sich bei der Wahrnehmung aller Aufgaben auf die ihnen sowohl durch das Grundgesetz (Art. 28 Abs. 2) wie durch die Landesverfassungen (etwa Art. 11 Abs. 2 BV) eingeräumte Selbstverwaltungsgarantie stützen. Sie dürfen diese Aufgaben eigenverantwortlich wahrnehmen.

Allerdings gilt dafür eine wichtige Einschränkung: Die Selbstverwaltung besteht nur im Rahmen der gesetzlichen Vorgaben. Zwar müssen diese wiederum verfassungsgemäß sein und auch die Rechtsstellung der Kommunen respektieren. Ein unbedingter rechtlicher Schutz wird dadurch aber nur für den Kernbereich der kommunalen Selbstverwaltung vermittelt. ${ }^{36}$

Tatsächlich ist jedenfalls die Freiheit der Kommunen, zu erledigende Aufgaben auszusuchen, weitgehend beschränkt. Viele sozialstaatliche Aufgaben im eigenen Wirkungskreis sind Pflichtaufgaben. Andere Aufgaben werden ihnen durch den Staat übertragen. Insbesondere die Sozialgesetzbücher setzen für die Gewährung von Sozialleistungen auch enge inhaltliche, also das „Wie“ der Aufgabenwahrnehmung betreffende gesetzliche Vorgaben. Der Gesetzgeber mißtraut, ob zu Recht oder Unrecht, zu großen Gestaltungsspielräumen und setzt zumeist auf einheitliche Standards. Zwar folgt aus der Selbstverwaltungsgarantie, daß Kommunen Spielräume zur freien Gestaltung innerhalb der Aufgaben des eigenen Wirkungskreises verbleiben müssen. ${ }^{37}$ Jedoch sind die Ausgaben für freiwillige Selbstverwaltungsaufgaben nach groben Schätzungen von ca. $20 \%$ zu Beginn der 1960er Jahre, über $10 \%$ in den 1970er Jahren auf ca. $5 \%$ zur Jahrtausendwende hin zurückgegangen. 38

\section{b) Zersplitterung kommunaler Aufgabenwahrnehmung}

Der kommunalen Selbstverwaltung ist eine weitere Begrenzung immanent, nämlich eine räumliche. Auch Kommunen haben Hoheitsgebiete, ihr Handeln ist grundsätzlich in dessen Wirkung auf diese Gebiete beschränkt. Das bedeutet zunächst, daß Selbstverwaltungsspielräume zu Unterschieden in der Ausgestaltung der Inklusion von einer Kommune zur nächsten führen können. Diese territorialen Ungleichbehandlungen sind durch den Gleichheitssatz nicht aufzufangen. ${ }^{39}$ Sie haben aber auf einer vergleichbaren räumlichen Ebene, also zwischen einzelnen Gemeinden, nur eine eingeschränkte praktische Bedeutung. Denn die eben genannten bundesgesetzlichen Vorgaben wirken insofern unitarisierend.

Wichtiger ist deshalb der Umstand, daß jedenfalls die deutschen Flächenstaaten über mehrere, wenn auch unterschiedlich ausgebaute kommunale Ebenen verfügen und die jeweilige Beschränkung der kommunalen Hoheitsgewalt im Zusammenspiel mit der

36 Grundl. BVerfGE 79, 127, 146 ff. (Rastede).

37 Tettinger/Schwarz, in: v. Mangoldt/Klein/Starck (Hrsg.), GG, Bd. 2, 6. Aufl. 2010, Art. 28 Rdnr. 247 ff. m.w.N.

38 Lohse, Kommunale Aufgaben, kommunaler Finanzausgleich und Konnexitätsprinzip, 2005, S. 52 f.

39 Henkel, Die Kommunalisierung von Staatsaufgaben, 2010, S. 212 ff. m.w.N. 
Selbstverwaltung verhindern, daß zwischen diesen Ebenen eine hierarchische Gliederung besteht. Einfacher ausgedrückt kann der überörtliche Träger den örtlichen Träger nicht verwaltungsintern anleiten und lenken. ${ }^{40} \mathrm{Da}$ aber gerade in den Materien der kommunalen Sozialpolitik zwei kommunale Ebenen die Verwaltungszuständigkeiten untereinander aufteilen, kommt es zu Koordinierungsproblemen.

So sind in Bayern (gem. Art. 82 Abs. 1 BayAGSG i.V.m. § 3 Abs. 3 SGB VIII) die Bezirke als überörtliche Träger der Sozialhilfe im eigenen Wirkungskreis für die Eingliederungshilfe für behinderte Menschen - immerhin seit 2008 in vollem Umfang ${ }^{41}$-, für alle übrigen Leistungen der Sozialhilfe, die in stationären bzw. teilstationären Einrichtungen gewährt werden, sowie für die Blindenhilfe gem. § 72 SGB XII zuständig, während Landkreise und kreisfreie Gemeinden als örtliche Träger der Jugendhilfe (gem. Art. 15 Abs. 1 S. 1 BayAGSG) zuständig sind für die erwähnten Leistungen nach § 35a SGB VIII. Auch wenn sich die Bezirke (gem. Art. 51 Abs. 1 S. 1 BayAGSG) an den Kosten der Eingliederungshilfe für seelisch behinderte Kinder und Jugendliche zu beteiligen haben, führt diese Regelung in der Praxis zu zahlreichen Kostenstreitigkeiten zwischen Bezirken und örtlichen Jugendhilfeträgern.

Von solchen Aufgabenüberschneidungen und Schnittstellenproblemen - zu denen auch die notwendige Abstimmung mit anderen Behörden, insbesondere den Pflegekassen im Hinblick auf die Versorgung von pflegebedürftigen Menschen, zählt - wird im Laufe der Tagung sicher noch mehrfach die Rede sein. Denn die Sozialraumperspektive macht, darin liegt gerade ein Vorteil, die aus einem Nebeneinander verschiedener Maßnahmen und zuständiger Träger folgenden Schwierigkeiten sichtbar und drängt auf deren Lösung. Die Kommunen sind sich dessen zunehmend bewußt. Erst jüngst hat darauf und auf ein von ihm initiiertes Modellprojekt beispielsweise der Bezirk Oberbayern in seinem ersten Bericht über eine strukturelle Gesamtplanung hingewiesen. ${ }^{42}$

\section{Die Finanzierung sozialstaatlicher Aufgaben}

\section{a) Grundsätze}

Die Erfüllung sozialpolitischer Aufgaben kostet Geld. Sie ist für die Kommunen mit steigenden Lasten verbunden, was angesichts der genannten Entwicklungen nicht ver-

40 Vgl. nur Becker, in: ders./Heckmann/Kempen/Manssen, Öffentliches Recht in Bayern, 5. Aufl. 2011, S. 76, Rdnr. 16.

41 Mit Inkrafttreten des Zweiten Gesetzes zur Änderung des Gesetzes zur Ausführung der Sozialgesetze vom 20.12.2007, GVB1., S. 979 zum 1.1.2008.

42 Erster Sozialbericht des Bezirks Oberbayern, abrufbar unter http://www.bezirk-oberbayern.de/media/custom/379_3575_1.PDF?1291866617; Wissel/Rohrmann, Örtliche Angebots- und Teilhabeplanung im Landkreis Weilheim-Schongau - Analysen, Einschätzungen, und Empfehlungen des Zentrums für Planung und Evaluation Sozialer Dienste der Universität Siegen, 2010, abrufbar auf http://www.uni-siegen.de/zpe/projekte/teilhabeplanung-wm/pdf/thp_wm_26112010.pdf. 
wundert. Um nur einige Zahlen zu nennen: Die Ausgaben für die Kinder- und Jugendhilfe stiegen bundesweit von 17,1 Mrd. $€$ im Jahr 2001 auf 24,3 Mrd. € im Jahr 2009; die für die Eingliederungshilfe für behinderte Menschen (6. Kapitel SGB XII) von 10,9 Mrd. $€$ im Jahr 2003 auf 13,8 Mrd. $€$ im Jahr 2010.43 Aber auch prozentual nehmen die Ausgaben für Sozialleistungen und soziale Einrichtungen wie Sozialhilfe, Kindergärten, Jugendhilfe, Alten-, Pflege- und Behinderteneinrichtungen einen wachsenden Anteil ein. Beliefen sie sich z.B. bei den Kommunen in Baden-Württemberg im Jahr 1980 noch auf $16 \%$ der Gesamtausgaben, so waren es im Jahr 1990 bereits $22 \%$ und im Jahr 2007 dann $30 \% .44$ Die Aufwendungen für die Ermöglichung eines barrierefreien $\mathrm{Zu}-$ gangs zu kommunalen Einrichtungen sind in diesen Zahlen natürlich noch nicht berücksichtigt.

Kommunen besitzen zwar mit eigenen Steuern, vor allem der Grund- und Gewerbesteuer, ihren Anteilen an staatlichen Steuern sowie den Beitrags- und Gebühreneinnahmen eigene Einnahmequellen, bleiben aber zu einem großen Teil auf staatliche Finanzmittel angewiesen. Das wichtigste Instrument zur Verteilung der Mittel stellen die kommunalen Finanzausgleiche dar. Mit ihnen wird Geld aus Steuerverbünden und dem Landeshaushalt auf die Kommunen, aber im Wege der interkommunalen Umlagen auch zwischen kommunalen Ebenen verteilt, wobei neben dem fiskalischen Ziel der Sicherung finanzieller Leistungsfähigkeit Ausgleichseffekte erzielt werden sollen.

$\mathrm{Da} ß$ trotz dieses Ausgleichs durch die zunehmenden Aufgaben im Zusammenhang mit der Gewährung von Sozialleistungen die Leistungsfähigkeit der Kommunen gefährdet werden kann, ist ein häufig vorgebrachtes Argument. Auf Bundesebene wurde auch deshalb im Jahr 1994 Art. 28 Abs. 2 GG um einen S. 3, Hs. 1 ergänzt. ${ }^{45}$ Danach umfaßt die kommunale Selbstverwaltungsgarantie ,die Grundlagen der finanziellen Eigenverantwortung". Damit wird das Erfordernis einer angemessenen Finanzausstattung ${ }^{46}$, das in der Rechtsprechung vieler Verfassungsgerichte der Länder anerkannt ist, ${ }^{47} \mathrm{im}$ Grundgesetz ausdrücklich verankert. Die Bestimmung verleiht den Kommunen aber keine praktisch handhabbare Abwehrposition. ${ }^{48}$ Sie haben wegen der Zweistufigkeit der Finanzverfassung keine unmittelbaren Ansprüche gegen den Bund, Sonderfälle wie den Belastungsausgleich des SGB II ausgenommen.

43 Quelle jeweils Statistisches Bundesamt, https://www.destatis.de (abgerufen am 13.6.2012).

44 Finanzministerium Baden-Württemberg (Hrsg.), Die Gemeinden und ihre Finanzen, 2010, S. 7.

45 42. Gesetz zur Änderung des GG v. 27.10.1994 (BGBl. I 1994, S. 3146).

46 Dazu nur BVerwGE 106, 280, 287. Vgl. auch den Überblick bei Laier, Der kommunale Finanzausgleich, 2008, S. 65 f.

47 Vgl. etwa StGHBW, ESVGH 49, 242; BayVGH, BayVBl. 2008, 172, 175 f.; LVerfG M-V E 17, 297, 318; Nds. StGH E 3, 136, 164; VerfGH NRW, NWVBl. 2008, 223 ff.; VerfGH Rhld-Pf, AS 29, 75, 82; VGH Saarl, AS RP-SL 34, 1 ff.; ThürVGRspr 2005, 233. Vgl. zur Berücksichtigung von Aufgabenerweiterungen VerfGH NRW, OVGE 43, 216.

48 Rennert, Die verfassungsrechtliche Stellung der Gemeinden bei der Zuweisung überörtlicher Aufgaben durch Bundesgesetz, VerwArch 94 (2003), S. 459, 468 ff. 


\section{b) Aufgabenübertragungsverbot und Konnexitätsprinzip}

Daran hat sich durch die Föderalismusreform I nichts geändert. Trotz vorangehender Diskussion wurde ein allgemeiner finanzieller Durchgriff von Bund zu Kommunen weiterhin abgelehnt und statt dessen ein Schutz der Kommunen durch ein Aufgabenübertragungsverbot (Art. 84 Abs. 1 S. 7 GG) eingeführt. Jetzt heißt es im Grundgesetz: „Durch Bundesgesetz dürfen Gemeinden und Gemeindeverbänden Aufgaben nicht übertragen werden." So klar das klingt, so unklar ist die Handhabung. Wichtig wäre das Verbot in erster Linie für Aufgabenänderungen. Ob es diese überhaupt erfaßt, ist umstritten. ${ }^{49}$ Das BVerfG hat dazu noch nie entschieden, wenn es das vielleicht auch demnächst muß. Greift der Schutz, so die Idee, dann müssen die Länder die Aufgabenübertragung vornehmen und dafür nach dem mittlerweile in allen Landesverfassungen vorgesehenen Konnexitätsgrundsatz ${ }^{50}$ einstehen: Denn danach soll zahlen, wer anschafft. Auch das klingt besser, als es möglicherweise ist. Hat nämlich der Bund seine Aufgabenzuweisung aus einem Sozialleistungsgesetz gestrichen und ändert er erst später den Inhalt der Aufgabe mit der Folge einer steigenden Aufgabenlast, so muß das Land dafür nur einstehen, wenn es einen eigenen Zurechnungsakt setzt. Läßt es seinerseits eine schon bestehende Aufgabenübertragung unverändert, so soll es nach verbreiteter Ansicht an einer solchen Zurechnung fehlen. 51

Aus diesem Dilemma gibt es für die Kommunen rechtlich gesehen 52 nur zwei Auswege: Entweder es gelingt eine Auslegung des zweistufigen Schutzmechanismus, mit der dessen Löchrigkeit geflickt wird. ${ }^{53}$ Oder es werden zumindest im Rahmen des kommunalen Finanzausgleichs die bundesrechtlich verursachten Mehrausgaben in die

49 Ausführlich dazu Becker, Der Schutz der Kommunen vor Aufgabenänderungen - Zu Aufgabenübertragungsverbot und Konnexitätsgebot am Beispiel des Bildungs- und Teilhabepakets und des Vormundschaftsänderungsgesetzes, Gutachten im Auftrag der kommunalen Spitzenverbände NRW, Materialien des Deutschen Städtetags 2012.

50 Erste Regelung in Art. 71 Abs. 3 LV BW v. 11.11.1953; letzte Regelung Art. 57 Abs. 4 Nds. Verf., eingeführt durch Gesetz zur Änderung der Nds. Verf. und des Gesetzes über den Staatsgerichtshof v. 27.1.2006 (Nds. GVBl., S. 58), rückwirkend in Kraft getreten zum 1.1.2006. Eine chronologische Darstellung der Einführung von Konnexitätsregelungen in den Landesverfassungen findet sich bei Henneke, Die Kommunen in der Finanzverfassung des Bundes und der Länder, 4. Aufl. 2008, S. $139 \mathrm{ff}$.

51 Engelken, Wenn der Bund seine alten Aufgabenzuweisungen an Kommunen aufhebt, DÖV 2011, S. 745, 750 f.; Engelbrecht, „Große Erwartungen“ - das „Kinderkrippen“-Urteil des VerfGH NRW vom 12.10.2010 und seine Auswirkungen für die bayerischen Kommunen, BayVB1. 2011, S. 718, $720 \mathrm{f}$.

52 Die dritte Lösung besteht in einer politischen Verhandlung über eine Kostenübernahme durch den Bund, vgl. etwa zum Beispiel der Finanzierung der Eingliederungshilfe durch den Bund als ,politische Gegenleistung" für die Zustimmung der Länder im Bundesrat zum Europäischen Fiskalpakt http://www.zeit.de/politik/deutschland/2012-06/fiskalpakt-bund-laender, abgerufen am 24.7.2012.

53 Vorschlag bei Becker, Der Schutz der Kommunen vor Aufgabenänderungen (Fußn. 49), S. 61 ff. 
Rechnung eingestellt, wie es der VerfGH Rhld.-Pfalz in einer jüngst ergangenen Entscheidung gefordert hat ${ }^{54}$ - wobei allerdings eine genaue Gegenrechnung nicht erfolgt.

\section{Schlu $\beta$}

Eine Sozialraumperspektive eröffnet - gerade im Zusammenhang mit einer umfassenden Querschnittsaufgabe wie der Inklusion von Menschen mit Behinderungen - einen Blick auf das vielfältige und oft schwierige Zusammenspiel unterschiedlichster hoheitlicher Maßnahmen.

Auch wenn räumliche Bezüge verschieden gegliedert sein können, unterstreicht diese Perspektive zugleich die wieder zunehmende Bedeutung einer kommunalen Aufgabenwahrnehmung, sowohl bezogen auf die Gewährung von Sozialleistungen als auch die Bereitstellung von Dienstleistungen und Einrichtungen der allgemeineren Daseinsvorsorge.

Diese Aufgabenwahrnehmung bleibt im Rahmen der deutschen verfassungsrechtlichen Ordnung komplex. Sie erfordert vor dem Hintergrund der Trennung von Bundesund Landesstaatsgewalt und der dadurch erschwerten Finanzbeziehungen die Abstimmung zwischen Bund, Ländern und Kommunen, zwischen verschiedenen kommunalen Ebenen, zwischen Kommunen und anderen Behörden sowie zwischen kommunalen Trägern und dritten Leistungserbringern.

Daraus resultieren vielfältige Herausforderungen bei der Erreichung des Ziels, allen Menschen auch in einem materiellen Sinn gleiche Teilhabechancen zu gewährleisten. Diese konnten und sollten hier nur angedeutet werden. Ihre nähere Herausarbeitung aus verschiedenen Blickwinkeln ist Gegenstand der in diesem Band im Folgenden abgedruckten Beiträge. 


\title{
Überall und nirgendwo - \\ „Disability Mainstreaming“ im kommunalen Lebensraum und Sozialraumorientierung als Transformationskonzept
}

\author{
Elisabeth Wacker
}

I. Nirgendwo - die (Un-)Sichtbarkeit von Behinderung und der Lebenschancenansatz

II. Sozialraum und ICF - Ansatzpunkte zur Transformation eines Lebensraums für alle

III. Überall - „Mainstreaming Disability“ als Entwicklungsprogramm und -methode

IV. Ausblick - evidenzbasierte Maßstäbe für Leistungssysteme als Königsweg?

V. Literatur

Die Veränderung kommunaler Praktiken im Umgang mit Personen mit Beeinträchtigung und Benachteiligung lohnt grundlegend neuer sozialwissenschaftlicher Aufmerksamkeit, auch wenn oder gerade weil geradlinige Prozesse des Wandels schwer aufzuzeigen sind. Vielmehr liegen - wie der Wissenschaftshistoriker Rheinberger ${ }^{1}$ dies beschreibt - wie in einem Bergwerk Goldadern nicht offen, sondern müssen entdeckt werden. Zwar stehen Wege in Form bereits gegrabener Tunnel und Schächte den Suchenden zur Verfügung, diese sind aber teilweise verwirrend angeordnet und Sicherheit, zielführend unterwegs zu sein, ist nicht einfach zu erlangen. Deswegen mag ein multidisziplinärer Blick zunächst als Verwirrung erscheinen, aber er kann auch als VerheiBung gedeutet werden, der Gefahr eindimensionaler Pfadfindung aus spezifisch fachlicher Perspektive zu entrinnen.

Wenn die Frage lautet, wie im Sozialraum Neues entsteht nach der Maßgabe einer aktuellen Vision der inklusiven Gesellschaft, so kann es verschiedene fachliche Tunnel und methodische Schächte geben, um dem Kern des Wissens näher zu kommen, das im Licht der Behindertenrechtskonvention der Vereinten Nationen (UN-BRK) ${ }^{2}$ neu zu betrachten ist. Der hier abgesteckte „Claim“ bezieht sich auf einen noch utopischen Le-

1 Rheinberger, Man weiß nicht genau, was man nicht weiß. Über die Kunst, das Unbekannte zu erforschen, NZZ vom 5.5.2007.

2 Das Übereinkommen der Vereinten Nationen vom 13. Dezember 2006 über die Rechte von Menschen mit Behinderung sowie das Fakultativprotokoll zum Übereinkommen sind in Deutschland seit 2009 geltendes Recht in Form einfachen Bundesrechts (BGBl. II 2008, S. 1419 ff.). 